\title{
MATRIMONIO SIN HIJOS: UN ESTUDIO DE CASO
}

Jesús Hanco Torres, Lic. Facultad de Teología Universidad Peruana Unión jesushanco@teologia.edu.pe

Fecha de recepción: Mayo 2012 Fecha de aceptación y versión final: Julio 2012

\section{Resumen}

El objetivo de esta investigación fue identificar los factores que influyeron para que una par eja de esposos decida no tener hijos. La presente investigación aborda el estudio de caso de una par eja de esposos, cuy o proyecto de vida incluía no tener hijos por más de 5 años. La información base se obtuvo a través de una serie de entrevistas, observaciones en el com portamiento de la pareja y mediante la realización de un grupo de discusión con la misma. Los factores identificados fueron los siguientes: (1) la búsqueda de la optimización del nivel de comprensión entre los miembros de la par eja; (2) el fortalecimiento del nivel económico de la pareja; y (3) el logro de sueños profesionales de la pareja.

Palabras clave: Pareja, familia, hijos, estudio de caso. 
Los hijos son la herencia del Señor, y somos responsables ante él por el manejo de su propiedad....

Elena G. de White, 1952

\section{Introducción}

Dentro de los últimos años se ha podido apr eciar en dif erentes países un alt o crecimiento de matrimonios sin hijos, situación que puede ser clasif icada, por lo menos debido a tr es factores: (a) inf ertilidad reproductiva de uno de los cónyuges, lo cual imposibilita la concepción de un hijo, ${ }^{1}$ (b) la postergación voluntaria del embarazo y (c) la abstención reproductiva permanente, siendo el mayor motivo de esta decisión, llevar una vida

1. Aproximadamente el $15 \%$ de parejas que desean tener bebés son incapaces de hacerlo, después de 1 año de relaciones maritales normales. La infertilidad obedece a diversas razones: problemas y descomposiciones hormonales, anormalidades genéticas e incompatibilidades inmunológicas, todas estas son razones que no dependen de la decisión de la persona y por otro lado, también ciertas enfermedades de transmisión sexual como la gonorrea y clamydea. Véase en Gary P. Stewart, Preguntas básicas sobre sexualidad y tecnología reproductiva (Grand Rapids: Editorial Portavoz, 2000), 12. más placentera, y libre de la responsabilidad de los hijos. Cabe recordar que dentro de este último grupo se hallan los denominados DINKYs . ${ }^{2}$ Durante los últimos años se ha visto en la sociedad, una cantidad creciente de matrimonios que van postergando la procreación. Aunque esto ha af ectado grandemente a diversos países desarr ollados como: EE.UU., Reino Unido, China, etc. Dentro de los es tudios realizados en Latinoamérica, se ha encontrado que las familias que no cuentan con hijos, se concentran en el quintil superior de ingr esos. ${ }^{3}$ Sin embargo, en países como el $\mathrm{P}$ erú, no se

2. DINKY, es un acrónimo que significa "Doble sueldo sin hijos aún" (Dual Income Not Kids Yet), es un término empleado en países como EE.UU., Reino Unido, Canadá y China para describir una pareja que tiene un alto ingreso, por no contar con hijos. Esto hace que la pareja sea capaz de permitirse un estilo de vida más caro, que los que tienen familias. El término fue acuñado en la década de los 80 en pleno auge de la cultura "Yuppie". Dinky, http://www.urbandictionary.com/define.php?term=dink. Para un mayor estudio véase Wouter van Gils y Gerbert Kraaykamp, The Emergence of Dual-Earner Couples. A Longitudinal Study of the Netherlands, in: International Sociology, Jg. 23, 2008, p. 345-366. 
ha registrado un estudio de caso sobre matrimonios que por decisión propia hayan elegido no tener hijos.

Desde la per spectiva constitucional, esta situación par eciera estar en contra del mismo concepto de "familia" y "matrimonio", que dentro de la constitución política del P erú son considerados como "ins titutos naturales y fundament ales de la sociedad". ${ }^{4}$ En es te caso, es base de la sociedad por la misma presencia de los hijos. P or otro lado, desde la per spectiva bíblica, el matrimonio tiene su

106 autor en el Cr eador del universo, en el jar dín del Edén (Gen 2:24) y "cuando se r econocen y obedecen los principios divinos en esta materia, el matrimonio es una bendición: salvaguarda la felicidad y la pur eza de la raza, satisface las necesidades sociales del hombre y eleva su naturaleza

3. Naciones Unidas, Panorama social de América Latina (Santiago de Chile: Publicación de la Naciones Unidas, 2004), 205.

4. Congreso constituyente democrático, Constitución Política del Perú 1993 (Lima. Red Ediciones, 2011), 12. física, intelectual y moral" ${ }^{5}$, por lo cual basado en la Biblia se puede argumentar que "el hogar es el corazón de la sociedad, de la iglesia y de la nación". ${ }^{6}$

Dentro del hogar, a través de la Biblia se puede apr eciar que la presencia de los hijos es fundamental, como lo menciona el Salmo: "herencia de Jehová son los hijos; cosa de estima el fruto del vientre" (Sal 127:3), a es to se añade la orden de Dios, para la reciente pareja establecida en el Edén: "Fructificad y multiplicaos; llenad la tierra, y sojuzgadla, y señor ead en los peces del mar, en las a ves de los cielos, y en todas las bestias que se mueven sobre la tierra" (Gen 1:28). De manera que la presencia de hijos dentro del matrimonio a través de la Biblia es considerada como una bendición y her encia de Jehová.

Por otro lado la ausencia de hijos en un hogar era considerado como un cas tigo de Dios

5. Elena G. de White, Patriarcas y Profetas (Buenos Aires: ACES, 1980), 25.

6. Elena G. de White, Hogar cristiano (Buenos Aires: ACES, 1986), 11. 
y por ende causaba af licción al matrimonio (Gen 20.18; 30:1,2). Aunque no se registra dentro de la Biblia el caso de una par eja que por acuerdo propio decida no tener hijos. Asimismo, Elena $\mathrm{G}$ de White extiende una exhortación a un matrimonio que no tiene hijos acerca del peligro que ronda sobre ellos:

Una casa sin hijos es un lugar desolado. El corazón de quienes la habitan corre peligro de volverse egoísta, de amar su propia comodidad y de consult ar sus propios deseos y con veniencia. Procuran simpatía para si, pero tienen poco que conceder a otros. ${ }^{7}$

Además de poder caer en diversos tipos de enf ermedad como lo declara White en Testimonios para la iglesia:

Muchos enferman física, ment al y moralmente porque dedican su atención casi e xclusivamente a si mismos. Podría salvarles del estancamiento la sana vit alidad de espiritus más jóvenes y diversos así como la inq uieta energía de los niños. ${ }^{8}$

\section{Ibid., 141.}

8. Elena G. de White, Testimonies for the Church (Michigan: Pacific Press Publishing Association, 2002), 2:647.
Por lo tanto, la figura de una familia completa es la q ue presenta a los esposos en compañía de sus hijos, siendo la ausencia de estos últimos, un mo tivo de extrañeza social. P or lo cual, el artículo consta de cuatr o preguntas realizadas a la joven pareja (5 años de casados). Al $\mathrm{f}$ inal de cada respuesta se añade un comentario del aut or investigador, esperando de es ta manera poder aportar a la visualización de factores que ocasionaron que un matrimonio cristiano no eligiese tener hijos por un periodo de tiempo.

\section{Descripción de la pareja en estudio}

Juan', es el ma yor de 3 her manos, su madre es ama de casa y su padre es comerciante. Por su lado María, es la tercera de cinco hermanos, cuya madre es ama de casa y su padr e es constructor. Ambos pr ovienen de hogares cristianos, y después de pasar por un periodo de amistad, pasaron por un periodo de 3 años de no viazgo al cabo del cual se casaron. Respecto al trabajo de Juan, durante sus 2 pri-

9. Los nombres reales han sido variados por motivos éticos. 
meros años de casado, se desarrollaron en una zona rural del país, siendo el ritmo de trabajo: 3 días fuera de casa (trabajo con comunidades rurales), y o tros 3 días en trabajos dentr o de una pequeña ciudad donde ambos vivían. Al cabo de dic ho periodo, Juan es trasladado del ár ea de trabajo hacia la capit al del país, donde se encuentran por más de 3 años.

\section{Entrevista con la pareja en estudio}

La presente recogida de datos, se ha esq uematizado de la siguiente manera: (a) pr egunta formulada a la par eja, (b) $r$ espuesta de la pareja, se han unido las apreciaciones de ambos, pues en la mayor parte coinciden en sus posiciones y (c) coment ario del autor basado en la declaración de la par eja y en apr eciación al modo cómo $r$ esponden a la pregunta.

Pregunta 1: ¿Cómo se sienten al estar casados por más de 5 años y no tener hijos?

En ocasiones un poco e xtraño, pero por lo gener al nos sentimos bien. Tal vez nos sentimos extranos, cuando nos encontramos en las reuniones interfamiliares, con diferentes familias de amigos y parientes contemporáneos. Donde casi toda la pr eocupación del matrimonio se encuentra circunscrita en sus hijos, su cuidado, sus estudios, su salud y ha\$a sus anécdotas. De manera que todo tema de conversación interfamiliar en dichas reuniones gira en torno de ellos, quizá más entre las esposas que entre los esposos. Sin embargo, nos sentimos bien cuando nos toca salir a algún lugar o cuando recibimos alguna invitación laboral, académica, deportiva, social o religiosa, que no in volucre la presencia de los niños.

Tal parece que la pareja siente la ausencia de tener hijos solo al encontrarse con otras parejas contemporáneas. Pero cuando ellos se hallan en sus correspondientes quehaceres y responsabilidades: estudios, trabajo, etc. Pareciera ser que son velados y no logran percibir la importancia de un hogar con hijos.

Pregunta 2: ¿Cómo percibieron la actitud de las personas ante la decisión de no tener hijos?

Tal vez nos hemos acostumbrado a frases como: "¿por qué demoran tanto sus hijos?", "¿cuándo llega 
la alegria a tu hogar?", "no sean egoístas", "tu jardin está sin flores", "si sigues demorando no vas a disfrutar a tus hijos", "cuando los quieras tener ya no tendrás fuerzas", y muc has otras frases más. Por una parte nos ha causado una cierta gracia, pero por otro lado hemos percibido una cier ta presión social desde los padr es hasta los amigos más allegados, quienes se hallan muy interesados en que tengamos hijos lo más antes posible.

Mientras veo que otras tienen algunas dificultades, creo que en mi caso tengo una ventaja. Entiendo pues, que tener hijos in volucra una responsabilidad paterna, la cual demanda un tiem po adicional para: jugar con ellos, cuidarlos durante un tramo del dia, escucharlos o auxiliarlos.

Aunque responden sonriéndose en un inicio, conforme van hablando se muestra su malestar por la presión social desde sus padres hasta sus "amigos más allegados" y la insistencia en ver que ellos tengan ya sus hijos. Por otra parte, ellos tratan de contrapesar esa presión con su declaración: "mientras veo que otros tienen dificultades", dando a entrever que las dificultades de otras parejas son un alivio para ellos, al parecer esas situaciones en cierto modo alimentan su decisión de postergar la tenencia de hijos.

Pregunta 3: ¿Cuáles han sido los motivos por los cuales han pasado cinco años sin tener hijos?

Sin duda, nuestro caso fue planificado en un comienzo pero luego se tornó cir cunstancial. Bueno, cuando recién nos casamos, colocamos el proyecto de esperar 2 años antes de tener hijos, creyendo asi poder disfrut ar nuestra vida en pareja y adaptarnos mejor el uno al o tro. A esta conclusión llegamos al poder ent ender que los dos primeros años son los que determinan la adaptabilidad de una pareja para el resto de la vida conyugal. De manera que esperamos dedicar esos 2 años para disfrutar nuestro matrimonio, saliendo juntos y tratando de comprendernos mejor como pareja.

$Y$ sin duda, los primeros años fueron bastante críticos debido a que ambos teniamos diferentes modos de ver las cosas, gustos, hábitos. Ante lo cual, cr eimos por conveniente esperar que pasen esos meses de adaptación hasta lograr 
comprendernos mejor. Quizá la idea que más consider amos es que al final era mi esposa(o), con quien voy a pasar el $r$ esto de mi vida.

Sin embargo, luego de los 2 años y cuando y a nos disponíamos a tener nuestros hijos, se presentó la oportunidad de poder continuar estudios de especialización por hallarnos en la capit al del pais. Por lo cual, decidimos pos tergar la llegada de los hijos hasta poder finalizar cada uno de nuestros estudios. Por otro lado, este periodo nos ha servido para poder consolidar la economía de nuestro hogar, pues ambos estamos estudiando y trabajando a la vez. Lo cual ha hecho posible de poder ir adq uiriendo algunos bienes necesarios para un hogar y también determinar un apoyo económico a nuestros padres con una fr ecuencia mensual. Y también contar con algunos ahorros, para la llegada de nuestro hijo(a).

Esta es la pr egunta más importante y el objetivo de la presente investigación, por lo cual, se ha tomado con sumo cuidado la declaración de la pareja.

Siendo el primer $f$ actor: la búsqueda de la optimización del ni- vel de comprensión entre los miembros de la par eja. Dentro de este factor, se puede ver que la pareja ha pasado por dif icultades durante sus primer os meses de casados, en los cuales de manera fortuita el no tener hijos hizo posible que tuviesen más tiempo para dedicarse el uno al otro. Otro aspecto, es la creencia que tiene la pareja respecto a que se puede disfrutar mejor del matrimonio, sin la pr esencia de los hijos, lo cual se podría deber a la percepción de que tener hijos no es una experiencia deseable, o puede ser esta una evidencia a cierto temor por parte de la pareja hacia la r esponsabilidad de tener hijos.

El segundo factor corresponde al logro de sueños pr ofesionales de la pareja. Sobre este factor, llama la atención que no estuvo presente desde un principio en la pareja. Sino por el contrario ellos tenían la seguridad de tener sus hijos luego del periodo de 2 años. Tal parece que la influencia del medio en el cual se desenvolvían al cabo de los dos años, jugó un papel muy im portante en ambos, de modo que el crecimiento académico y pr ofesional fue priorizado a la tenencia de hijos. Por otro lado, también 
esto se podría deber a la falta de realización profesional de la pareja (o de uno de sus miembros), punto no considerado antes de casarse.

El tercer factor corresponde al fortalecimiento del nivel económico de la pareja, este factor aunque es mencionado al final, es donde la pareja coloca más argumentos a su favor: (a) bienes necesarios para su hogar, (b) apoyo económico para sus padr es y (c) ahorros para la llegada de su hijo. Llama la atención, la manera cómo presentan estos argumentos, pues parecieran ser un escudo que protege su decisión de no tener hijos.

Pregunta 4: Después de estos cinco años de espera ¿Ha cambiado en algo su per spectiva de tener hijos? ¿Cuál es su apr eciación actual sobre tener hijos?

Creemos que nuestra perspectiva sobre los hijos no ha cambiado, aunque no descar tariamos que nos estemos acostumbrando a vivir sin hijos. Pero nuestra perspectiva siempre fue que los hijos son herencia de Jehová, por ende una gran responsabilidad para la pareja y deben ser frutos del amor y no del descuido. Asimismo, cr eemos que es una equivocación que la pareja trabaje y estudie mientras tienen sus hijos peq ueños, menores a 7 años (v arón), y hasta en la adolescencia (dama). U no de los cónyuges debe estar dispuesto y realizar un sacrificio para dejar de trabajar y dedicarse de lleno a sus hijos. Y sobr e tenerlos, creemos que ya es tiempo de tenerlos.

Aunque ellos aseveran que su perspectiva no ha cambiado, se puede visualizar que sí ha sufrido una variación, en su declaración "nos estemos acostumbrando a vivir sin hijos". De alguna manera el hecho de estar más de cinco años sin hijos está haciendo que la pareja crea que eso es normal. Por otra parte, ellos es tán en contra de un hogar donde ambos trabajen y a la $\mathrm{v}$ ez eduquen a sus hijos, por lo menos respecto a los menor es de sie te años desde la perspectiva del esposo. Aunque la esposa argument a que es en la adolescencia, donde la presencia continua de uno de los padres es también importante. Y finalmente, aunque ambos no tienen hijos, no descar tan la posibilidad de poder tenerlos en un futuro muy cercano. 


\section{Conclusión}

Luego de realizar la entrevista a la pareja de esposos cristianos, se puede concluir que los factores que influenciaron para que ellos decidieran no tener hijos por más de cinco años fuer on los siguientes: (1) la búsq ueda de la op timización del nivel de comprensión entre los miembros de la pareja; (2) el fortalecimiento del nivel económico de la pareja; y (3) el logro de sueños profesionales de la pareja.

Se infiere de esta manera que dichos factores están influenciando para que otras parejas tomen la decisión de pos tergar la procreación de los hijos. Es un desafío para una in vestigación subsiguiente poder contras tar estos factores con la cosmo visión bíblica de hogar, de acuerdo al modelo divino. 